THE PROBLEM OF ABUNDANCES FOR "METAL-RICH" GLOBULAR CLUSTERS

Bengt Gustafsson

Astronomical Observatory, Uppsala, Sweden

\title{
ABSTRACT
}

The discrepancy between the results of recent high-dispersion analyses and of studies at lower resolution for the classical metal-rich globular clusters is discussed and different explanations for this discrepancy are considered. It is concluded that further systematic investigations are motivated in order to establish the more or less interesting astrophysical explanations for it.

\section{WHAT IS THE PROBLEM?}

For a long time, the most metal-rich globular clusters, such as 47 Tuc and $M 71$, have been thought to bridge the gap in age and metalenrichment between the extreme Pop. II objects and those of the disc of our galaxy. In recent years, however, this view has been questioned. On rather good grounds, drastic revisions of the more or less generally accepted metal abundances of these clusters have been suggested. The situation is illustrated in Fig. 1, where published metal-abundance estimates for 47 Tuc and $M 71$ are plotted as a function of time. Analyses of essentially the same observational material are connected with lines. As a comparison, a similar diagram is also shown for the more metal-poor cluster $M 13$.

In Fig. 1 the crosses represent determinations of metal abundances based on photometry or low-dispersion spectrograms of single stars. These determinations exploit a number of different methods; such as the use of ultraviolet excesses and other photometric metal-abundance criteria, calibrated by model-atmosphere calculations or less sophisticated techniques. Also included here are some estimates from spectra at relatively low resolution, sometimes calibrated with synthetic spectra.

The dots in Fig. 1 denote spectroscopic studies at high resolution. Such studies for the metal-rich clusters have become feasible due to the efficient echelle spectrographs at the big telescopes at Kitt Peak and 101

Richard M. West (ed.), Highlights of Astronomy, Vol. 6, 101-108.

Copyright $\odot 1983$ by the IAU. 


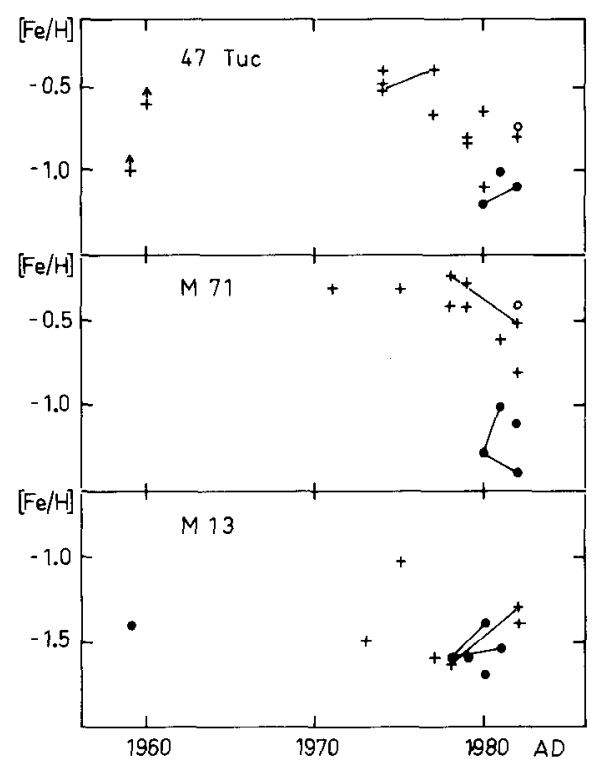

Figure 1. Different estimates of metal abundances of stars in three globular clusters, plotted as a function of time of publication. Studies based on photometry and low/moderate dispersion spectroscopy are represented by crosses, results of high-dispersion analyses are denoted by dots. The open circles refer to Bessell's (1982) recent high-dispersion analyses. Lines connect results based on essentially identical observational material.

Cerro Tololo and the efforts of Judith Cohen, Catherine Pilachowski and some other astronomers. For $M 13$ results from work at rather high resolution were available as early as 1959 , thanks to the classical study of Helfer, Wallerstein and Greenstein (1959), based on $5 \mathrm{~m}$ coudé spectra of one giant in the cluster. In addition, these authors established an [Fe/H] value for the very metal-poor cluster $M 92$ which is also close to recent determinations.

It should be noted that the high-resolution results for 47 Tuc and M 71 by Pilachowski, Canterna and Wallerstein (1980) and Cohen (1980), respectively, have now been confirmed by other high-dispersion analyses (Cottrell and Da Costa, 1981; Pilachowski, Sneden and Wallerstein, 1982). However, when the preparation of the present paper was essentially completed, Bessell (1982) reported high-dispersion results (denoted by open circles in Fig. 1). These deviating results will be discussed in the last section, below.

IS THERE A PROBLEM?

The problem is now whether any problem is left. The cynic might argue 
that Fig. 1 is a nice illustration of the so-called "sheep effect" - the scientist (at least the male scientist) tends to follow his colleagues as long as his methods are not accurate enough to force him to choose truth instead of being staunchly loyal to his colleagues. For some representative metal-poor clusters we were fortunate enough to get good abundance determinations very early - no wonder that the more approximate and indirect methods later verified those results. For the most metalrich clusters, however, this fix point was not there - the shepherd was absent and the herd went astray.

A careful reading of the literature on the metal abundances of 47 Tuc and $M 71$ shows that this interesting explanation is not totally out of place. E.g., in some studies during the seventies there were indications that the clusters should be more metal poor than previously thought, but these results were sometimes explained away, more or less elegantly, or just not given the proper weight by the authors themselves or their colleagues ( $c f$. studies by Hesser, Hartwick and McClure, 1977; Gustafsson and Ardeberg, 1978; Mallia, 1978). The moral may be: If you want to discover anything which is unexpected by you and your colleagues, you should have significantly better methods than they have. Otherwise they, and you, will not believe you.

But this is certainly not the most important explanation for the discrepancy between crosses and dots in $\mathrm{Fig}$. 1. The $[\mathrm{Fe} / \mathrm{H}]$ values denoted by crosses tend to decrease already before the dots appear in the upper panels - indeed, a significant revision of the metal abundance of 47 Tuc was first suggested on the basis of uvby photometry (Dickens, Bell and Gustafsson, 1979). Moreover, the crosses represent many different, and sometimes independent approaches. The reasons why most of them deviate from the high-dispersion results could be worth studying, in order to learn more about the properties or low-dispersion spectroscopy or even more about the stars themselves. Also, even the most accurate contemporary chemical analysis at high resolution of stars may be severely affected by systematic errors which are hard to control or estimate or even to think of. The globular cluster stars are apparently faint, of about the 12 th magnitude or fainter, and high dispersion analyses of such stars requires not only hard scientific work but also some artistery and even sometimes a little magic. Therefore, there is still this annoying feeling that the metal abundances of 47 Tuc and $M 71$ are considerably more uncertain than the estimates for clusters where the different determinations agree reasonably well, such as the case for $M 13$ and other more metal-poor clusters.

\section{SOLUTIONS?}

Several workers have tried to clarify the situation and explain the discrepancies between the different methods for obtaining metal abundances of the cluster stars. This discussion is interesting but rather technical, and therefore I can only give a very schematic sketch of it below. Those who are interested in more details should consult the 
proceedings of the Joint Scientific Meeting of the IAU Commissions 25, 29 37 and 45 at the XVIIIth General Assembly. These proceedings will be published in the P.A.S.P. At that meeting, the systematic errors in abundance analyses of metal-poor stars were scrutinized.

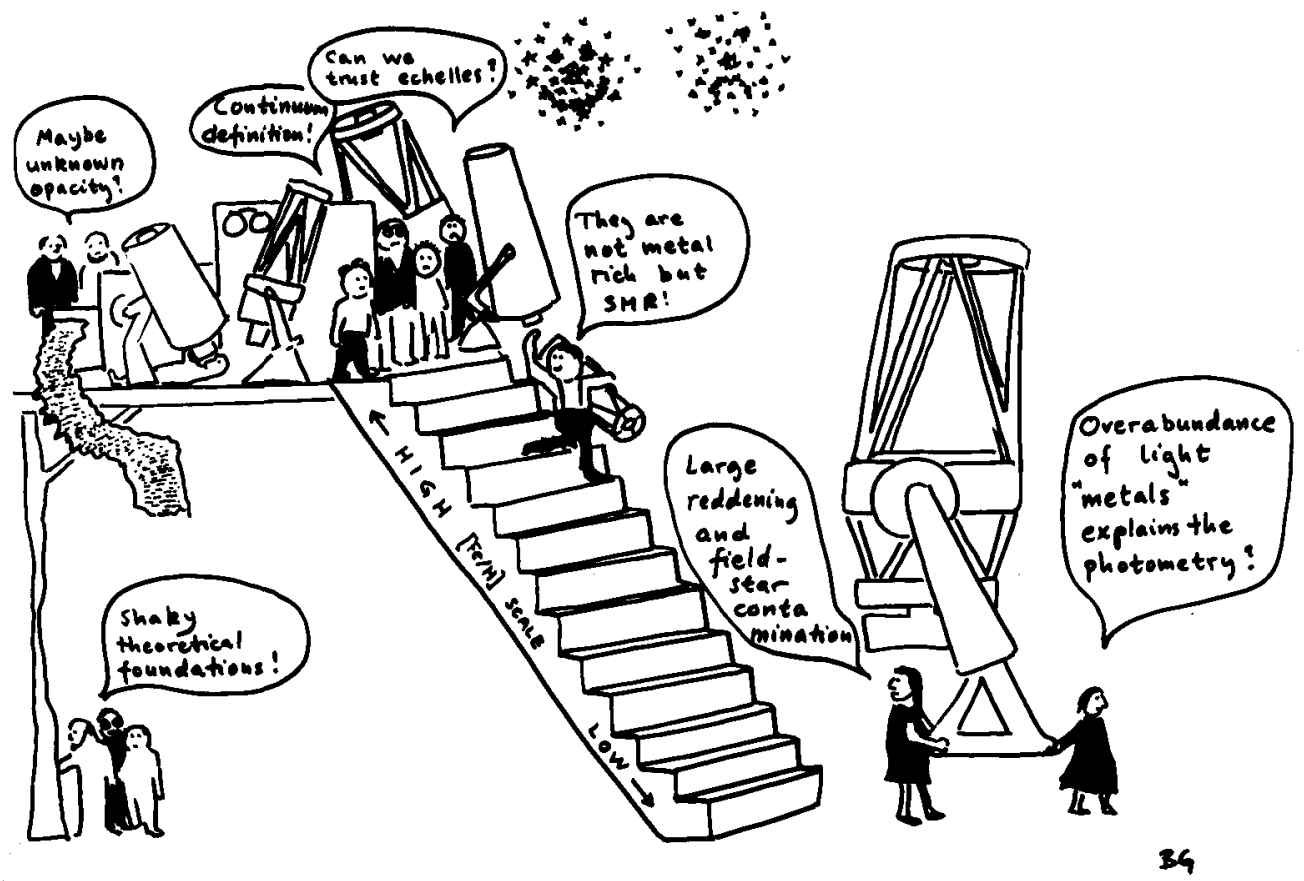

Figure 2. Do they dare to follow the ladies?

What, then, are the suggestions for resolving the discrepancy? (Some of these are illustrated in Fig. 2.) Naturally, methodological errors have been discussed. Suggestions that the equivalent widths measured in echelle spectra are systematically wrong have been made ( $\mathrm{Bell}, 1982$; $\mathrm{Bell}$ and Gustafsson, 1982a) and contested (Pilachowski et al., 1982, cf., however, Bessell, 1982). The effects of the large reddening and the obvious risk for field-star contamination for the photometry of $M 71$ have been stressed (Cohen, 1980) but some arguments against this explanation have also been presented (Bell and Gustafsson, 1982a). Accurate narrowband photometry should be obtained for the cluster stars studied spectroscopically, in order to rule out some of this possible source of error.

As usual, the fundamental problems in model-atmosphere analyses of stars have been brought up by several groups. This is reasonable, since the classical models and their spectra are founded on a basis of dubious assumptions - the plane-parallel stratification, the neglect of probably important hydrodynamic phenomena, the assumption of Local Thermodynamic Equilibrium. In the past, these assumptions were often justified ad posteriori, by referring to the consistency obtained in abundance 
analyses based on these assumptions. Now, when we face inconsistencies which look significant, we certainly ought to worry, not least since the stars investigated have quite low surface gravities and have few, if any, equals in the solar neighbourhood where the problems could be studied more thoroughly. This situation requires more theoretical and observational work on the general problems of stellar atmospheres. At present, we can only say that departures from LTE and from plane-parallel stratification, inhomogeneities and other hydrodynamic phenomena may well affect the spectra of globular cluster stars significantly and differently from the effects on the Sun and other comparison stars in the solar neighbourhood. This is even more serious since such effects are thought to be more dangerous for the saturated spectral lines, and such lines are important in all present analyses (cf. Gustafsson, 1982). From this point of view it is very important to measure more weak lines for cluster stars with higher atmospheric pressures, i.e., fainter stars. This requires more time at larger telescopes - which these problems deserve.

This has brought us into the more astrophysical explanations for the different abundance results for the metal-rich globular clusters. It seems probable that the discrepancy should not be swept under the carpet since it may tell us something about the extent to which the real stars are different from what we think (or pretend) them to be.

One explanation, which has essentially been brought forward by Catherine Pilachowski and collaborators (1980, 1982a), is based on the fact that the stars of 47 Tuc and $M 71$ have been found in the highdispersion analyses to be richer in light elements relative to iron than is the Sun. Therefore, it is not clear that it is meaningful to compare the overall metal abundance determined from photometry - with different elements weighted differently for different photometric systems - with the cleaner iron abundances $[\mathrm{Fe} / \mathrm{H}]$ determined from spectroscopy. The bands of the photometric metal indices, like $\delta$ (U-B), the usual metal-abundance indicators of the narrow-band DDO photometry and the Searle and Zinn blocking index $\mathrm{S}$, all contain lines from such species as $\mathrm{CN}$, ionized $\mathrm{Ca}$ and neutral Ti. According to Pilachowski et al. this overabundance of elements lighter than iron might explain the seemingly high overall metal abundance derived from photometry. Roger Bell and I, however, have looked closer at this possibility by calculating synthetic spectra and colours and find that the spectroscopically derived overabundances of light elements do not all seem sufficient to cause such dramatic effects on the stellar colours as those observed (Bell and Gustafsson, 1982b and c). This situation seems to be the same for the TiO measurements by Jeremy Mould and collaborators; the photometry suggests a significantly higher abundance of titanium and/or oxygen for $M 71$ than the high-dispersion analyses indicate ( $\mathrm{c} f$. Johnson, Mould and Bernat, 1982 and papers cited therein); however, for 47 Tuc the agreement may be better (Mould and Bessell, 1982). For certain photometric calibrations, notably that of the Searle and Zinn system, the effective-temperature vs. gravity relation adopted for the giant branch is of vital importance (cf. Bell and Gustafsson, 1982b). However, this relation was found by Rood (1981) to be highly invariant to CNO abundance variations, assuming that the mixing 
length parameter does not vary systematically with these abundances. We do not know how valid this latter assumption is.

Another possible explanation for the discrepancy is that the theoretical calculations of colours, which form an important part in the calibrations of the photometric systems in present use, fail to consider all important opacity sources. We have earlier traced the need for such an unknown opacity source in the solar ultraviolet spectrum (Gustafsson et al., 1975; Gustafsson and Bell, 1979) and in the uv spectra of red giants. Later, we traced effects of this opacity in the blue-violet spectrum of Arcturus (Frisk et al., 1982), as well, and its presence has recently been demonstrated for other stars of intermediate Pop. II (Bell and Gustafsson, 1982c). We estimate that if the theoretical calibration of the photometry is corrected for the effect of this unknown opacity source, the metal abundance from the Searle and Zinn photometry should be reduced by a factor of two or possibly even three for the "metal-rich" clusters while the calibration for the metal-poor clusters is unaffected. So, this may be half of the explanation we are looking for.

What is the nature of this opacity source? A good candidate is a veil or haze of very weak metal lines that are not included in the extensive line lists that are used in the synthetic-spectrum calculations. Systematic photometry for stars with accurately determined chemical abundances might help in revealing the true nature of the source. From this point of view it is interesting to see the discordant properties of the $M 71$ stars and other cluster giants in the Searle and Zinn system, recently pointed out by Pilachowski, Sneden and Wallerstein (1982a).

Still another possibility, suggested by McClure and Hesser (1982), is that the globular cluster stars may be affected by phenomena similar to those of so-called super-metal-rich (SMR) stars of Pop. I. McClure and Hesser, as well as Cohen et al. (1980) and Canterna, Harris and Ferrall (1982) have discussed moderate resolution spectra of individual stars in several globular clusters. They find that strong absorption features are significantly stronger in 47 Tuc and $M 71$ stars than in traditionally more metal-poor clusters such as $M 5$. Yet, with the new spectroscopic abundance scale 47 Tuc and $M 71$ should not be more metal rich than the other clusters. The phenomenon, which occurs not only for the strong lines of the light elements but also for strong lines of elements at the iron peak, resembles the case of the notorious super-metal-rich stars as compared with more normal Pop. I stars. One possible, though not necessarily probable, explanation for the spectra of SMR stars is that the outer layers of the photospheres are cooled, compared with normal stars, by some unknown process (cf. Desikachary and Gray, 1978 and Deming, 1980). Such a cooling would certainly also affect the photometry, causing the stars to look more metal-rich than they really are. If the analogy between the problem of metal-rich clusters and that of SMR stars is relevant, we have learned that the SMR phenomenon probably is pre-stellar in origin, since it seems to affect all the giants in the cluster. Thus, it would be reasonable to assume its origin to be related to abnormal abundances of some element(s) or, possibly, to the angular momentum of the stars. 
Further accurate abundance determinations, e.g. of the CNO elements and for stars further down along the giant branch, are necessary to investigate this possibility.

Whatever the reason for the strengthened strong lines may be, it is important to clarify it, since strong lines certainly will continue to be used as abundance indicators in studies of chemical evolution in external galaxies (cf., e.g., the recent study of globular cluster stars in the Large Magellanic Cloud by Cohen, 1982). It is also quite important to try to understand why the appearance and location of the giant branches are significantly different for the classical metal-rich clusters, as compared with the ones which are now claimed to have the same abundance of heavy elements (cf. Demarque, King and Diaz, 1982).

\section{CONCLUSION AND WARNING}

In conclusion, we have found that the reason(s) for the discrepancy between the metal abundances derived from high and low spectral resolution, respectively, for clusters like 47 Tuc and $M 71$ may well be more than non-trivial and well worth further studies. In particular, it would be interesting to know whether this discrepancy shows up not only for seemingly metal-rich clusters in the halo but also for those in the inner regions of the Galaxy. Additionally, there is an urgent need to understand the low-resolution abundance criteria that must be used in studies of considerably more distant objects.

However, before too much speculation and hard work is spent in further attempts to understand the deviating abundance results in terms of physical properties of the stars themselves, the possible errors in the high-dispersion analyses should be studied further. Bessell's (1982) very recent investigation of one giant in 47 Tuc and one in $M 71$, in a strictly differential procedure vs. Arcturus, is based on IPCS spectra at a resolution similar to that of the other high-dispersion analyses. His results, however, are very different: $[\mathrm{Fe} / \mathrm{H}]=-0.75$ and -0.4 for 47 Tuc and $M 71$, respectively. As is seen in Fig. 1 these results agree well with the photometric results. Bessell seems to ascribe much of the differences between the high-resolution studies to errors in the location of the continuum in the echelle spectra of the previous investigations. As a moderation of my previous stern moral it seems justified to add the following advice: Another way to make new discoveries may be to try not to forget previous results and methods just because the new ones are thought to be better in principle.

\section{ACKNOWLEDGEMENTS}

Mike Bessell, Judith Cohen, Jeremy Mould and Catherine Pilachowski are thanked for sending me preprints and other unpublished information and Roger Bell, Nils Bergwall, Kjell Eriksson and David Minugh for valuable comments on the manuscript. 


\section{REFERENCES}

Bell, R.A.: 1982, in "Astrophysical Parameters for Globular Clusters", eds. A.G.D. Philip and D.S. Hayes, L. Davis Press, Schenectady, p. 325.

Bell, R.A. and Gustafsson, B.: 1982a, Astrophys. J. 255, 122.

Bell, R.A. and Gustafsson, B.: 1982b, Monthly Notices Roy. Astron. Soc., in press.

Bell, R.A. and Gustafsson, B.: 1982c, in preparation.

Bessel, M.S.: 1982, preprint.

Canterna, R., Harris, W.E. and Ferrall, T.: 1982, preprint cited by Pilachowski, Sneden and Wallerstein (1982a).

Cohen, J.G.: 1980, Astrophys. J. 241, 981.

Cohen, J.G.: 1982 , Astrophys. J. $\overline{258}, 143$.

Cohen, J.G., Frogel, J.A., Persson, S.E. and Zinn, R.: 1980, Astrophys. J. 239, 74 .

Cottrell, P.L. and DaCosta, G.S.: 1981, Astrophys. J. (Letters) 245, L79.

Demarque, P., King, C.R. and Diaz, A.: 1982, Astrophys. J. 259, 154 .

Deming, D.: 1980, Astrophys. J. 236, 230 .

Desikachary, K. and Gray, D.F.: 1978, Astrophys. J. 226, 907 and ibid. 236, 1056.

Dickens, R.J., Bell, R.A. and Gustafsson, B.: 1979, Astrophys. J. 232, 428.

Frisk, U., Bell, R.A., Gustafsson, B., Nordh, H.L. and Olofsson, S.G.: 1982, Monthly Notices Roy. Astron. Soc. 199, 471.

Gustafsson, B.: 1982, submitted to Publ. Astron. Soc. Pacific.

Gustafsson, B. and Ardeberg, A.: 1978, in "Astronomical Papers dedicated to Bengt Strömgren", eds. A. Reiz and T. Andersen, Copenhagen Univ. Obs., p. 145.

Gustafsson, B. and Bell, R.A.: 1979, Astron. Astrophys. 74, 313.

Gustafsson, B., Bell, R.A., Eriksson, K. and Nordlund, A.: 1975, Astron. Astrophys. $42,407$.

Helfer, H.L., Wallerstein, G. and Greenstein, J.L.: 1959, Astrophys. J. 129,700 .

Hesser, J.E., Hartwick, F.D.A. and McClure, R.D.: 1977, Astrophys. J. Suppl. 33, 471 .

Johnson, H.R., Mould, J.R. and Bernat, A.P.: 1982, Astrophys. J. 258, 161. Mallia, E.A.: 1978, Astron. Astrophys. 70, 115.

McClure, R.D. and Hesser, J.E.: 1982, in "Astrophysical Parameters for Globular Clusters", eds. A.G.D. Philip and D.S. Hayes, L. Davis Press, Schenectady, p. 145.

Mould, J.R. and Bessell, M.S.: 1982, Astrophys. J., in press.

Pilachowski, C.A., Canterna, R. and Wallerstein, G.: 1980, Astrophys. J. (Letters), 235, L21.

Pilachowski, C.A., Sneden, C. and Wallerstein, G.: 1982a, preprint.

Pilachowski, C.A., Sneden, C., Dominy, J.F., Cottrell, P. and May, D.C.: $1982 \mathrm{~b}$, preprint.

Rood, R.T.: 1981, in "Physical Processes in Red Giants", eds. I. Iben Jr. and A. Renzini, D. Reidel Publ. Co., p. 51. 